\title{
Bolovac Application for HF and Microwave Power Measurement and Standardization
}

\author{
M. C. Selby* \\ Institute for Basic Standards, National Bureau of Standards, \\ Boulder, Colorado 80302
}

(August 25, 1970)

\begin{abstract}
The bolometric voltage and current (BOLOVAC) standard for frequencies to $18 \mathrm{GHz}$ and higher, recently developed at the National Bureau of Standards (NBS), can also be used to measure power, offering the following advantages over a number of other methods in use today:

(1) It eliminates:

(a) the uncertainty resulting from neglecting termination mismatch

(b) measurement of reflection coefficients and computations using complex equations

(c) "Limits-of-error" charts, thus rendering definitive results

(d) use of impedance charts

(e) dc or af calibrations of power meters

(f) need of corrections such as "effective efficiency" and "calibration factor."

(2) It covers a wide frequency range $(0.5 \mathrm{MHz}$ to $18 \mathrm{GHz}$ or wider)

(3) It should result in substantial reduction of calibration time in many instances

(4) It can be applied to calibrate feed-through power measurement methods for power levels ranging into kilowatts.

In measuring power, the Bolovac (1) measures the voltage across a known resistance component of a given load, or (2) measures the voltage in any plane of a coaxial line of known characteristic impedance and known voltage distribution along the line, or (3) absorbs the rf power as any other absorptiontype power mount does. In the first two cases resistance and voltage distribution measurements may be made separately or these measurements may be combined with the power measurement procedure. In the third case the rf power is absorbed in a special disk-type bolometric detector of the Bolovac and is equal to the dc- (or af) bias-power substituted in that detector; this bias power is measured with conventional power substitution bridges. Any appreciable unaccounted for power losses occur outside the Bolovac and can be determined employing conventional techniques as well as the Bolovac itself. The Bolovac needs no rf calibration. A Bolovac may have a power range of $20 \mathrm{~dB}$ or higher, depending on accuracy desired, and a maximum power approaching its safe power dissipation, e.g., $0.5 \mathrm{~W}$ or higher. This paper is limited to the application of the Bolovac to power measurements only and presents analytical and practical aspects of this application.
\end{abstract}

Key Words: Bolometric power standards; Bolovac power measurements; calibration of power meters; $\mathrm{RF}$ and microwave power measurements; standard of power through $18 \mathrm{GHz}$.

\section{Introduction}

The bolometric voltage and current (BOLOVAC) standard $[1]^{1}$ was developed originally to furnish accurate hf and microwave voltages and currents. It was the first successful attempt to extend the direct measurement of these quantities well into the microwave range $[18 \mathrm{GHz}$ or higher]. This device turns out to be as useful for power measurement and standard-

\footnotetext{
1 Figures in brackets indicate the literature references at the end of this paper

*Electromagnetics Division, National Bureau of Standards, Boulder, Colorado 80302
}

ization either as a feed-through or absorption type with apparent advantages over some of the widely used methods at present.

In measuring power, the Bolovac (1) measures the voltage across a known resistive component of a given load, or (2) measures the voltage in any plane of a coaxial line of known characteristic impedance and known voltage distribution along the line, or (3) absorbs the rf power as any other absorption-type power mount does. In the first two cases resistance and voltage distribution measurements may be made separately or these measurements may be combined with the power measurement procedure. In the third case 
the rf power is absorbed in a special disk-type bolometric detector of the Bolovac and is equal to the dc(or af) bias-power substituted in that detector; this bias power is measure with conventional power substitution bridges. Any appreciable unaccounted for power losses occur outside the Bolovac and can be determined employing conventional techniques as well as the Bolovac itself. Perhaps the major contribution of the Bolovac is in that it resolves the difficulty introduced by the most serious source of uncertainty in power measurement, namely, the mismatch error $[2,3]$. Directional couplers accomplish the same purpose but do so over a relatively narrow range of frequency for individual couplers. In addition, the directivity, the coupling factor and the side-arm power meters of each coupler must be calibrated. On the other hand, as will be seen below, the application of the Bolovac inherently eliminates the mismatch uncertainty, has a wide frequency range (e.g., $0.5 \mathrm{MHz}$ to $18 \mathrm{GHz}$ ), and needs no rf calibration.

The application of known rf voltages to power measurements introduces a number of other advantages, namely, the elimination of:

(1) measurement of power-meter correction factors, e.g., effective efficiency and calibration factor,

(2) measurement of source and load complex reflection coefficients,

(3) computation involving complex formulas containing these reflection coefficients,

(4) using "limits-of-error" charts as an alternative to measuring phase angles of reflection coefficients and

(5) complex computations or the application of impedance charts in measuring load impedances.

Basically the Bolovac measures voltages at all the frequencies mentioned and makes the above advantages possible. A brief description of the Bolovac is given later in the text. However, because the advantages of its application are listed at the outset in this introduction, it seems appropriate to list also its present limitations.

The relative difficulties and disadvantages encountered to date at the National Bureau of Standards (NBS) in the construction and application of the Bolovac are briefly as follows.

(1) The voltage sensor of the Bolovac is a split bolometric thin-film disk. Such disks do not seem available commercially. The NBS so far has succeeded in developing a fabrication technique for disk values up to about $10 \Omega ; 50-\Omega$ films were produced, but are not yet successfully assembled. These $50-\Omega$ disks are particularly desirable.

(2) Impedance measurements are needed to determine the resistive components of the loads connected to the Bolovac (this is not needed when the Bolovac is used as an absorption-type power meter). However, the text below shows that these impedance measurements do not involve phase measurements and do not require complex computations, or the use of complex impedance charts.

(3) Attention must as usual be given to such common difficulties as reproducibility and losses of connectors, stub tuners or slotted lines; the Bolovac, however, may be employed to determine such losses.

(4) As in all other cases of bolometric measurements, the application of the Bolovac requires a resistance bridge and a precalibrated dc- or af-biasing power source. The use of thermoelectric or photoemissive sensors [1,4] will still require de calibration, so that, these applications are essentially equivalent to dcpower substitution calorimetry.

Considering the above advantages and limitations, the application of the Bolovac holds promise for radical improvements in coaxial power measurements.

This paper deals with the application of the Bolovac to power measurements. Additional papers are planned to deal in detail with its other applications.

The ultimate objective in measuring $\mathrm{rf}$ or microwave power is to determine the net power flow from a given source to a given load with maximum possible accuracy. When the load is an intermediary tool, (e.g., an uncalibrated power meter of any kind), toward that ultimate objective, the operation may be referred to as "calibration." Fairly sophisticated calibration techniques are used at the NBS. These require

(1) high-qualtiy, relatively expensive, calorimetric, substitution or other standards,

(2) considerable space,

(3) personnel with proper technical qualifications,

(4) intercomparisons between independent and quasi-independent methods, and

(5) considerable time [3].

A basic difference between these methods and the one employing the Bolovac, is that none of the present methods measures rf voltages at frequencies above about $10 \mathrm{MHz}$. Another basic difference is that the Bolovac, when used as a power absorption device, has no dc-for-rf power substitution error and has essentially all of the net rf power flowing to the Bolovac absorbed in its bolometric disk; thus only de or af reference standards are needed to calibrate a power bridge employing a Bolovac as a mount. The major advantage in measuring power with the Bolovac, is the prospect of its application in facilites one or more calibration echelons removed from the NBS with little or no loss in the accuracy available at NBS. Two factors seem to make this possible:

(1) the previously mentioned elimination of complex reflection-coefficient measurements including associated relatively complex computations and

(2) elimination of accuracy degradation between calibration echelons.

Because the greatest uncertainties are introduced by the reflection coefficients (referred to as "mismatch errors") and because their elimination has been a major goal in past efforts to improve power measurements, these errors are discussed below at some length.

Calibration and measurement steps commonly used at present. The NBS calibrates at present two figures of merit for coaxial absorption type meters, namely:

$\eta_{e}=$ effective efficiency $=\frac{P_{\text {ind }}}{P_{n}}$ 
$K_{c}=$ calibration factor $=\frac{P_{\text {ind }}}{{ }_{f} P}=\eta_{e}\left(1-\left|\Gamma_{m}\right|^{2}\right)$

where

$P_{\text {ind }}=$ dial reading of the power meter which in many cases is the dc or af power substituted for $\mathrm{rf}$ power in the bolometers of a bolometric type meter.

$P_{n}=$ net power absorbed by the meter

$=$ incident power, ${ }_{f} P$, minus reflected power, ${ }_{r} P$.

$\Gamma_{m}=$ complex reflection coefficient of the power meter.

Uncertainties of about \pm 1 to 2 percent for $\eta_{e}$ and $K_{c}[3,7]$ are quoted by the NBS at frequencies to 18 GHz. Similar calibration terms are measured for feedthrough type meters with the same uncertainties for frequencies to $4 \mathrm{GHz}$ and \pm 2 to 3 percent for higher frequencies.

If a load with reflection coefficient, $\Gamma_{1}$, is substituted in a laboratory outside the NBS for a calibrated power meter having a reflection coefficient, $\Gamma_{m}^{\prime}$, connected to a generator with reflection coefficient, $\Gamma_{g}$, the relationship between the net power absorbed by the standard meter, $P_{n}^{\prime}$ and that absorbed by the load, $P_{1}$, is $[2,3,8]$.

$$
P_{1}=\frac{P_{\text {ind }}^{\prime}}{\eta_{e}^{\prime}}\left[\frac{1-\left|\Gamma_{1}\right|^{2}}{1-\left|\Gamma_{m}^{\prime}\right|^{2}} \cdot \frac{\left|1-\Gamma_{g} \Gamma_{m}^{\prime}\right|^{2}}{\left|1-\Gamma_{g} \Gamma_{1}\right|^{2}}\right]
$$

Here $P_{\text {ind }}^{\prime}$ is the indicated power on the calibrated meter with effective efficiency, $\eta_{e}^{\prime}$, and reflection coefficient $\Gamma_{m}^{\prime}$. Of course the load, itself, can be another power meter.

Two special cases are of particular interest:

(a) when the load matches the characteristic impedance, $Z_{0}$, of the line, $\Gamma_{1}=0$, then the net power absorbed by the load, designated for this case as $P_{0}$, is given by

$$
P_{0}=\frac{P_{\text {ind }}^{\prime}}{\eta_{e}^{\prime}} \frac{\left|1-\Gamma_{g} \Gamma_{m}^{\prime}\right|^{2}}{1-\left|\Gamma_{m}^{\prime}\right|^{2}} .
$$

(b) When the load is a conjugate match of the generator impedance, $\Gamma_{1}=\Gamma_{g}^{*}$, then the net power, designated for this case as $P_{c}$, is given by

$$
P_{c}=P_{0} \frac{1}{\left(1-\left|\Gamma_{g}\right|^{2}\right)} .
$$

Examination of eqs (3) shows that both the magnitude and phase of the $\Gamma_{s}^{\prime}$ have a great effect on the accuracy of power measurements. It is not uncommon to have $|\Gamma|$ values as high as 0.2 to 0.5 (corresponding to VSWR, S, of 1.5 to 3 ) in the frequency range to 18 $\mathrm{GHz}$. Their phases may vary from zero to $\pm 180^{\circ}$. It is relatively simple to measure the magnitude $|\Gamma|$ (or S) of a load but more troublesome to measure its phase angle. It is even more difficult to measure the magnitude and phase of $\Gamma_{g}$, particularly when it must be made under operating conditions of the generator. The present common practice is to measure only the magnitudes of the $\Gamma_{s}^{\prime}$, to assume their phases to be zero, and to use eqs (3). The latter assumption introduces a serious uncertainty, sometimes referred to as "termination uncertainty." For example, when $\left|\Gamma_{1}\right|=0.2$ and $\left|\Gamma_{g}\right|=0.3$, the effect of neglecting their phases will introduce an error in $P_{n}$ that could be as high as 25 percent. Values of these worst-case uncertainties for various combinations of $\left|\Gamma_{1}\right|$ and $\left|\Gamma_{g}\right|$ are given in various published charts and monographs; however, these values can not be used to correct a given value of $P_{n}$. Only measured phase angles of the $\Gamma_{s}^{\prime}$ could make this possible. Unfortunately such measurements are seldom, if ever, made [3], particularly in measurement facilities outside the NBS and other top level laboratories. The logical conclusion is to use power-measurement methods that do not require the measurement of the phases of $\Gamma_{s}^{\prime}$. The Bolovac provides such a method. Moreover, the procedures available with the Bolovac make it possible to incorporate the measurement of the absolute magnitude of $\Gamma_{1}$ into a routine, relatively simple step of the power measurement procedure and, in addition, eliminate the need of measuring $\Gamma_{y}$ altogether. This will be discussed further.

The degradation of accuracy as standard power meters are calibrated in a series of echelons starting with a top standardization laboratory and ending with the field of operation is also of major concern. A degradation of 2,3 , or 4 to 1 in accuracy is generally prescribed between echelons $[8,10]$. Thus, for example, an uncertainty of 1 percent in the indication of a meter calibrated at the NBS may inflate into an uncertainty of 27 percent by the time it reaches the operational field through 3 intermediary calibration echelons [10], each having a degradation of 3:1. Use of the Bolovac (which does not require rf calibration) could eliminate this degradation of accuracy.

\section{Basic Principles. Physical and Electrical Characteristics of the Bolovac}

The Bolovac may be looked upon as an rf voltmeter measuring voltages in an orthogonal plane, (i.e., the only plane where voltages are defined) of a coaxial line operating in a TEM mode [1]. Transmission (including slotted) lines throughout this text are assumed to have negligible losses unless specified otherwise. Figure 1 shows the basic elements of the Bolovac connected through a transmission line to an rf source. These elements are (1) a bolometric thin-film disk orthogonally located in a coaxial assembly, (2) bias feed-through type terminals to connect this disk to a resistance bridge, (3) bias blocking capacitors built into the feed-through terminals, and (4) "input" and "output" connectors. The disk consists of a thin resistive annular film and electrodes connecting the film to the coaxial conductors. To prevent shorting the bias power, the outer conductor and the film of the disk are split along a diameter. The rf voltage, $V_{B}$, appearing across the thin film is determined con- 
ventionally by the bridge measuring the reduction of biasing power dissipated in the bolometric thin-film disk located in that plane. The two halves of the disk are connected in series for de and in parallel for $\mathrm{rf}$. The rf resistance of the disk is equal to its de resistance at all frequencies in question to 0.001 percent or better [1]. Thus, if the dc resistance of the disk is $200 \Omega$ per side, its rf resistance is $50 \Omega$. Providing there is sufficient rf signal to permit adequate bridge balancing, correct voltages are measured regardless of the impedance presented to the disk by the generator and the load. In its use the Bolovac disk is located within 0.001 to 0.002 in of the input plane of a device under test.

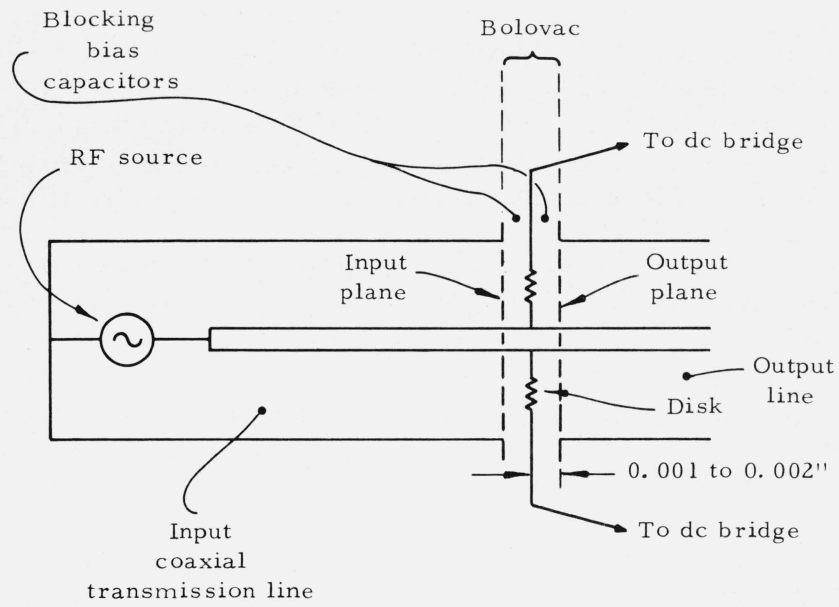

FigURE 1. Schematic diagram of Bolovac connected between input and output coaxial transmission lines.

The application of the Bolovac, as a voltage measuring device, to power measurement may be described as follows. Figure 2 shows a schematic presentation of a power meter or load $\mathrm{M}$ having its input at plane $m$. The equivalent lumped circuit admittance, $Y_{p}$, to the right into plane $m$ is shown as an equivalent resistance $R_{p}$ shunted by a reactance $X_{p}$. The net power absorbed by the load is

$$
P_{n}=V_{B} / R_{p}
$$

where $V_{B}$ is the voltage measured by the Bolovac at plane $m$. If the line is lossless, the same $V_{B}$ can be measured at any other plane $n \lambda / 2(n=$ integer $)$ away from plane $m$, e.g., plane $A$.

The value of $R_{p}$ may be measured in various ways, e.g., with a slotted line of negligible loss as shown in figure 3 , in which case it is given by

$$
R_{p}=\frac{V_{\max }}{V_{\min }} Z_{0}\left(V_{m} / V_{\max }\right)^{2}=S Z_{0}\left(V_{m} / V_{\max }\right)^{2}
$$

where

$Z_{0}=$ characteristic impedance of the slotted line $S=V_{\max } / V_{\min }$

$V_{m}=$ the voltage at plane $m$ or at a plane in the slotted line $n \lambda / 2$ away from plane $m$

$V_{\min }=$ is the voltage minimum in the line

$V_{\max }=$ is the voltage maximum in the line

The uncertainties in measuring $R_{p}$ and $P_{n}$, including the effect of slotted-line losses, are discussed below under error analysis.

Equation (5) derived in appendix A shows that the computation of $R_{p}$ does not involve complex values nor the aid of the Smith or other impedance charts. The accuracy of $R_{p}$ depends on the accuracy of $Z_{0}$ and of two rf voltage ratios. The state-of-the-art of impedance and $\mathrm{rf}$ voltage-ratio measurements permits relatively high accuracies [7].

When the Bolovac is used with an equal arm bridge (see appendix B), the value of the voltage appearing across $R_{p}$ is

$$
V_{B}=0.25\left(V_{0}^{2}-V_{1}^{2}\right)^{1 / 2}
$$

where $V_{c}$ and $V_{1}=$ dc-bias bridge voltages without and with $\mathrm{rf}$ respectively.

Appendix B gives other forms of eq (6).

Once $R_{p}$ is known, the load is connected directly to the Bolovac and, combining eqs (4), (5), and (6a), the net power flow into the load is given in terms of
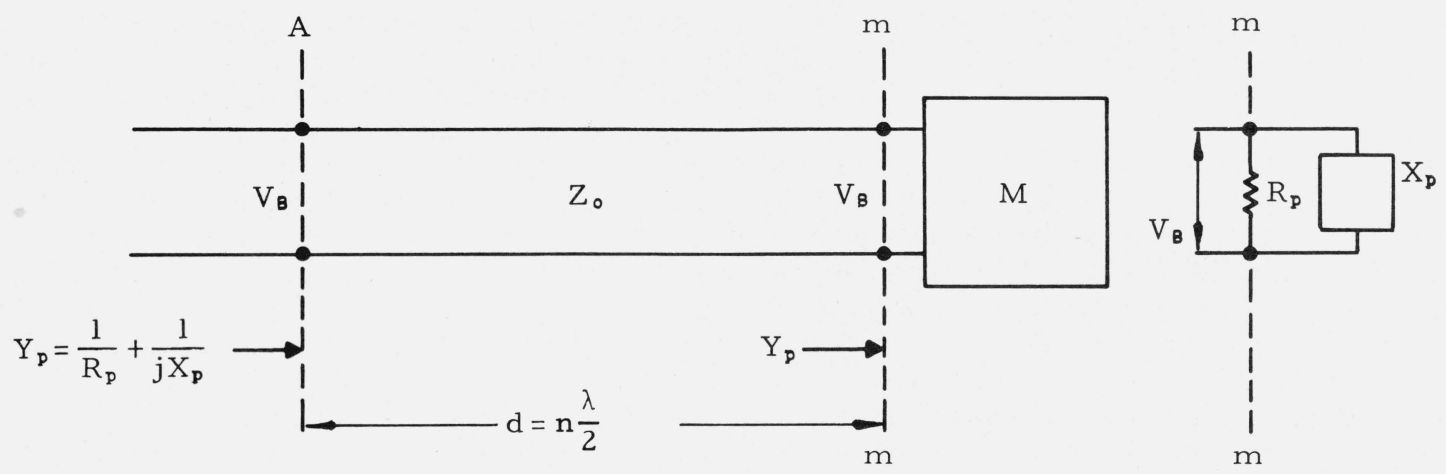

Figure 2. Bolovac connected to a power meter or load, $M$, directly (at plane $\mathrm{m}$ ) or through a lossless line (at plane A). 
$Z_{0}$, dc voltages, and ratios of rf voltages by

$$
P_{n}=\frac{\left(V_{B} \frac{V_{\max }}{V_{m}}\right)^{2}}{S Z_{0}}=\frac{1}{16 S Z_{0}} \quad\left(V_{0}^{2}-V_{1}^{2}\right)\left(V_{\max } / V_{m}\right)^{2} .
$$

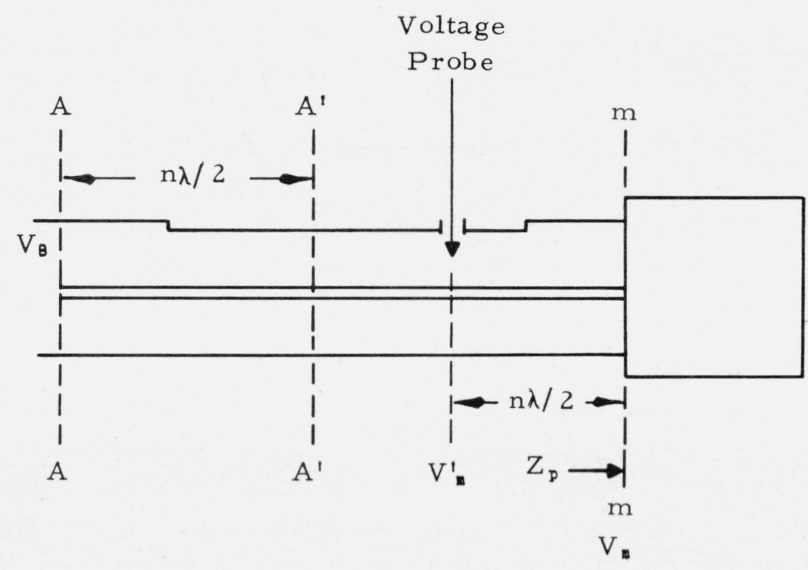

FigURE 3. Calibration of power meter with Bolovac and slotted line.

Since a lossless line is assumed, a simpler computation may be used in one step, i.e., leaving the slottedline between the Bolovac and the meter. In this case

$$
P_{n}=\left(V_{B}^{\prime} \frac{V_{\max }}{V_{B}^{\prime}}\right)^{2} / S Z_{0}
$$

where $V_{B}^{\prime}=$ the voltage in plane $A^{\prime}$, fig. $3, n \lambda / 2$ away from the Bolovac disk, assumed to be equal to $V_{B}$.

The Bolovac, its connector and the slotted lines are constructed to have the cross section of the line between $A$ and $A^{\prime}$ uniform.

Again, $V_{B}^{\prime}$ in eq (8) may be replaced by its equivalent in (6a) when an equal arm bridge is used with the Bolovac.

Thus, the Bolovac permits the measurement of the net power absorbed by the meter under calibration without the necessity of measuring "substitution" power, complex reflection coefficients, $\eta_{e}$, and $K_{c}$.

If for any reason, e.g., to check manufacturers' specifications it is desired to find the incident power, $\eta_{e}$, and $K_{c}$, eqs (1) and (2) may be used, recalling that

$$
{ }_{f} P=\frac{P_{n}}{1-\left|\Gamma_{m}\right|^{2}}=\frac{P_{n}}{1-\left(\frac{S-1}{S+1}\right)^{2}} .
$$

Because indicated or substituted bias power of the meter under test is not needed for its calibration by the Bolovac, one may dispose of the de or af calibration of the power meter. However, this presently required calibration may be desirable for secondary purposes, e.g., for uniformity in production and repair of the indicating or "instrument" part of power meters with- out the bolometer mounts. The instruments are then interchangeable with the same mount. When the meter is calibrated in terms of substituted bias power, eq (1) may be used to find $\eta_{e}$.

It may be of interest to note that eq (8) can be used to determine the rf voltage in a given plane of a coaxial system in terms of known power absorbed by a load. For example, a calibrated power meter (or a Bolovac used as a power meter) placed as a termination of a feed-through type sampling oscilloscope yields the net power flow past the input plane to the oscilloscope. The voltage in this plane is $V_{B}$. The ratios $S$ and $V_{\max } / V_{B}^{\prime}$ are measured in a slotted line terminated by the above sampling oscilloscope-Bolovac combination. However, one must keep in mind several sources of possible errors, namely, (1) discontinuities in the slotted line between the input plane and the plane in the slotted line Where $V_{B}^{\prime}$ is located, (2) losses between the $V_{B}^{\prime}$ plane and the power meter and (3) the correction factor, if any, of the power meter.

Direct application of the Bolovac as a source of voltage eliminates these errors and reduces the measurement time from hours to minutes per frequency.

\section{Applications}

\subsection{Measuring Net Power Flow in a Transmission Line With a Bolovac}

In practice one is generally interested in measuring the power flow in a transmission line under one of the three following impedance matching conditions:

(1) Maximum power available from a source (when a conjugate match exists along the line). This power will be designated as $P_{c}$.

(2) Power flow when there are no standing waves in the line, (i.e., when the source looks into a load having an impedance equal to the characteristic impedance of the line). This power will be designated as $P_{0}$.

(3) Net power flow, $P_{n}$, under any matching conditions including the above.

Measurement of $P_{c}$ and $P_{0}$ is usually of interest when one wishes to evaluate the power-delivery capabilities of a source. Measurement of $P_{n}$ is generally desired when one wishes to know the net power absorbed by the load. The latter, as indicated above, may be a power meter under calibration.

To determine $P_{c}$ or $P_{0}$ of an "unknown" source with the Bolovac, any power meter in series with a slotted line and a stub tuner is first used as a load. The input end of the slotted line is connected to the source, and the stub tuner is connected at the output end of the line. To measure $P_{c}$ the stub is adjusted for maximum indication of the power meter. To measure $P_{0}$, the stub tuner is adjusted for flat voltage distribution along the line. In either case the source is replaced by a Bolovac. The level of $V_{B}$ is adjusted to reproduce the respective meter reading. The value of $V_{B}, S$ and $V_{B} / V_{\max }$ are measured and eq (7), or equivalent, yields the value of $P_{c}$ or $P_{0}$. 


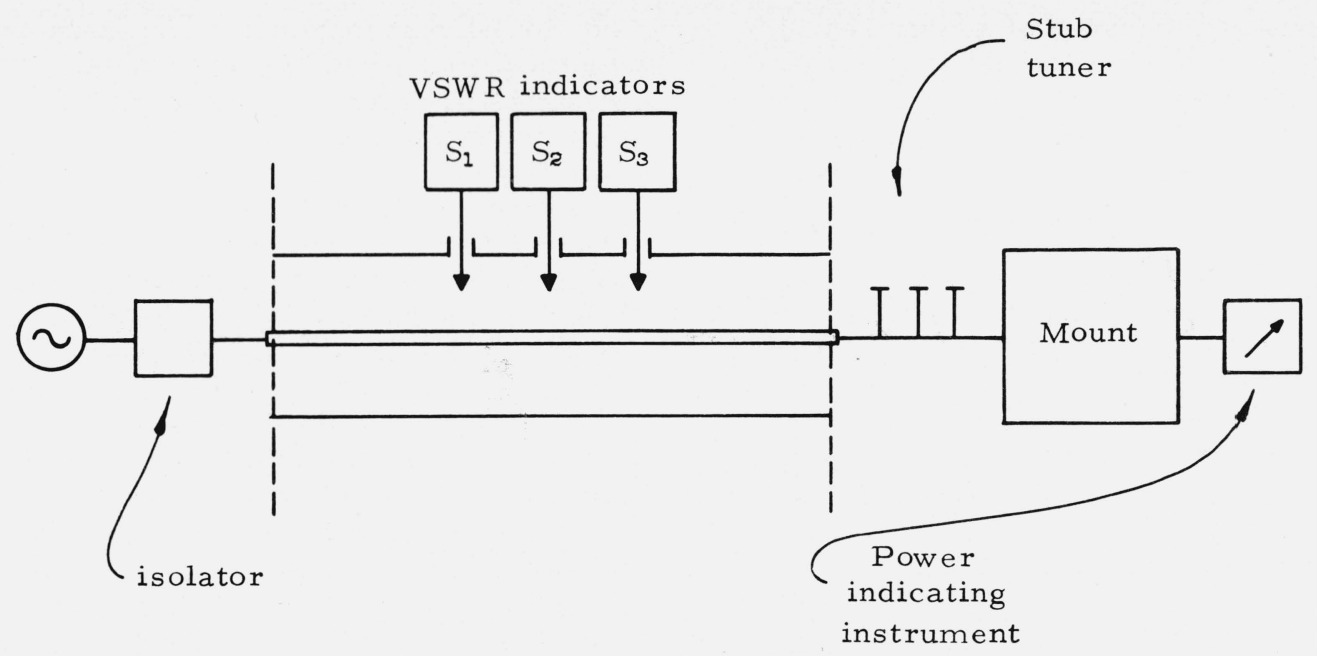

FiguRE 4. Setup to obtain a $\mathrm{Z}_{0}$-match of a power meter.

A relatively simple procedure is suggested in appendix $\mathrm{C}$ to accomplish the $Z_{0}$-match adjustment of the stub without resorting to the hitherto employed "hit or miss" time-consuming and tedious procedure. The price to pay for this relief is the employment of three temporary probes in the slotted line as described in the appendix and illustrated in figure 4.

\subsection{Monitoring Net-Power Flow in a Transmission Line}

The net power, $P_{n}$, may be computed using (7), provided (1) one voltage, $V_{B}$, is measured with the Bolovac at any plane in the line and (2) the ratios $S$ and $V_{\max } / V_{B}$ are measured. There are several ways to obtain $V_{B}$ and the above ratios. For example:

(1) A slotted line section with a probe precalibrated with a Bolovac [5] may be permanently installed in the system. A multiple probe (MP) line-section, discussed later may be used instead.

(2) A Bolovac plus the slotted or MP line section may be permanently installed in the system. This eliminates the need of probe calibration. However, it is desirable to have a Bolovac with a value of $R \gg Z_{0}$ to reduce the loading effect of the Bolovac to a minimum. The availability on the market of high-resistance thin thermistor films seems to make this feasible.

\subsection{Application of the Bolovac as a Load or as an Absorption Type Power Meter}

The Bolovac may be used as an absorption-type power meter as illustrated in figure 5 . For this purpose the Bolovac is turned end for end, so that its normally "output" side (fig 1) becomes its input side facing the generator end. A single sliding short is connected to the other (normally input) side of the Bolovac and is set for antiresonance at the specific frequency at which the measurement is made. It can be shown [11, 12, 13] that the antiresonant input impedance of a good quality $50-\Omega 7 \mathrm{~mm}$ coaxial sliding short will be of the order of $10,000 \Omega$ or higher at frequencies to $18 \mathrm{GHz}$. The input impedance of the Bolovac is then essentially one-fourth the equivalent dc resistance of the disk at any frequency under consideration here, for values of $R_{s}$ of $50 \Omega$ or lower.

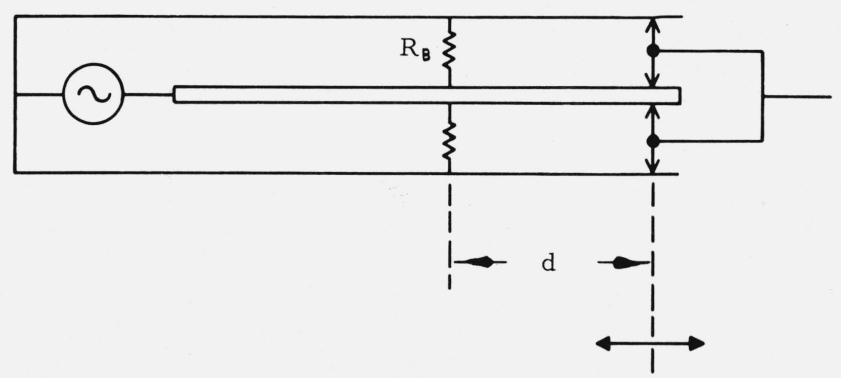

Figure 5. Application of the Bolovac as an absorption-type power meter.

Since $\eta_{e}$ for the Bolovac is unity, the net absorbed power is given by

$$
P_{n}=V_{B}^{2} / R_{B}^{\prime} \doteq V_{B}^{2} / R_{B}=P_{\mathrm{dc}}(\mathrm{sub})
$$

where $P_{\mathrm{dc}}(\mathrm{sub})=\mathrm{dc}$ power substituted in the Bolovac and $R_{B}^{\prime}=$ equivalent disk rf resistance shunted by the admittance of the sliding short. One may connect the Bolovac in series with a three-stub tuner to a source, adjust the tuner for maximum $V_{B}$ and find $P_{n}$. To find $P_{c}$ the losses in the tuner are added to $P_{n}$. For the case of $R_{B}=Z_{0}$ one achieves a zero reflection-coefficient power meter for the antiresonant frequencies of the sliding short. Moreover such a Bolovac with the sliding short constitutes a $Z_{0}$-termination over its entire frequency range to an accuracy limited by structural perfection of the disk. With other $R_{B}$ values the Bolovac makes available a standard mismatch which does not require rf calibration.

The antiresonant settings of the sliding short can be predetermined for a number of frequencies in order to obviate the necessity of making the adjust- 
ment each time the frequency is changed. Several such shorts will cover a wide frequency range. The mechanical setting of the short is not critical because the antiresonant impedance curve is relatively flat around its maximum. A procedure to determine an antiresonant position is to first find two adjacent resonant positions at which $V_{B}$ is minimum. The antiresonant position of the short is then located halfway between the two resonant positions. Once calibrated, the set of the sliding short is ready for use with any Bolovac at any time.

A power meter may be calibrated by substitution of a Bolovac operating in the power-absorption mode as illustrated in figure 5. This method seems particularly attractive because of its relative simplicity and because it does not need a slotted line. The procedure is as follows. The power meter is connected to a stable source in series with a three-stub tuner. The latter is adjusted for conjugate match, i.e., for maximum indication of the power meter. The meter is then replaced by the Bolovac and the stub tuner readjusted for maximum $V_{B}$ of the Bolovac. Since the maximum available power, $P_{c}$, from the source is generally constant, the indicated meter power is equal to $P_{\mathrm{dc}}$ (sub). Because the input $S$ value of the Bolovac might be quite different from that of the meter the tuner losses will not be the same and generally a small correction can be introduced for the difference.

It should be pointed out that the sliding short is not necessary for the measurement of $P_{n}$ with the Bolovac used as an absorption power meter. However, in this case the input resistive component, $R_{B}^{\prime}$, of the Bolovac (without the sliding short) has to be measured and computed with eq (5). $P_{n}$ is then given by eq (4) substituting $R_{B}^{\prime}$ for $R_{p}$.

\subsection{Application of the Bolovac for Feed-Through Power Measurement}

It was pointed out above that the major source of uncertainty in present-day absorption (as opposed to feed-through) type power measurements is the mismatch error introduced by the terminal reflection coefficients in the transmission line. One method of eliminating this difficulty is to use directional couplers. Because the Bolovac, like directional couplers, eliminates the need of measuring $\Gamma_{g}$ and $\Gamma_{1}$, its potential application for feed-through power measurement is briefly discussed here. It is assumed that the reader is familiar with directional couplers and will be in a position to assess the relative applicational merits of the couplers vis-a-vis the Bolovac.

A high resistance Bolovac, e.g., one with a disk resistance for example, 100 times $Z_{0}$, permanently placed in a transmission line, will continuously measure the voltage in a certain plane of the line. A probe in a slotted section of this line will furnish the voltage ratios required in eqs (7) and (8) to compute the net, incident, or reflected power flow in the line. With additional electronic instrumentation it may be possible to read these power components directly. Multiple-probe line sections may be used instead of slotted sections for fixed frequencies or limited frequency ranges. Application of MP lines for power and impedance measurements have been treated analytically in the past [14, $15,16,17,18]$ and need not be discussed here.

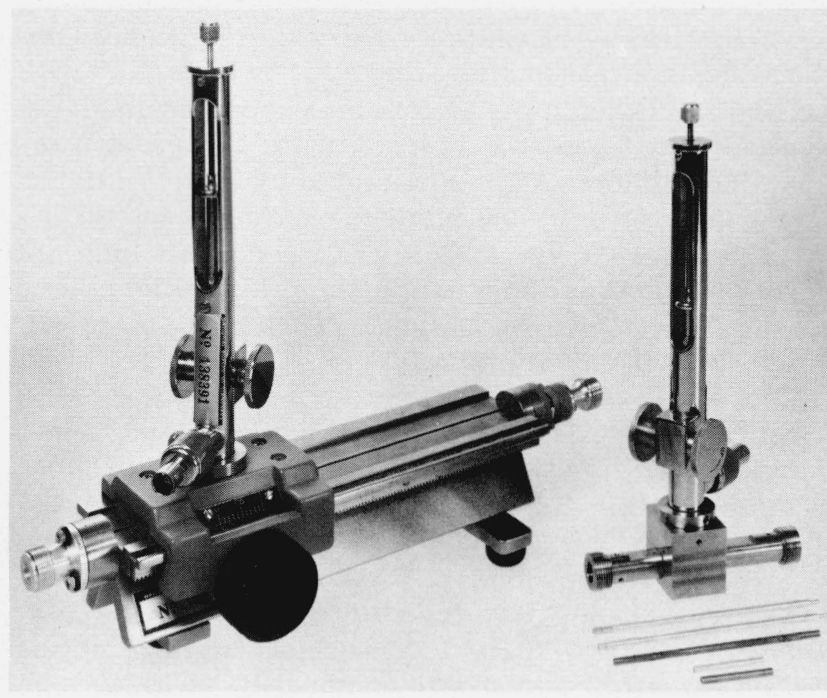

Figure 6. NBS-built multiple-probe (MP) line (right) and commercial slotted line are shown.

Commercial probes are used with both lines. Some of the self-supporting and end supported center conductors and connecting pins for the MP line are in the foreground.

Figure 6 shows an experimental NBS MP section in comparison with a commercial slotted line; the latter, though more expensive, has a range of 2 to $18 \mathrm{GHz}$ as compared to two fixed frequencies $(5$ and $10 \mathrm{GHz}$ ) useable with the MP line with equal accuracy. The frequency limitation of an MP line is a result of the requirement that the fixed probes be an eighth of a wavelength apart. Figure 7 shows the equivalent schematic diagram of an MP line and appendix D gives equations of immediate interest in its application. An MP line may be built with a number of probe holes located so that the above spacing is attainable at several harmonic frequencies, such as $1,2,4,8$ and 3,6 and $12 \mathrm{GHz}$. Thus approximately the same number of

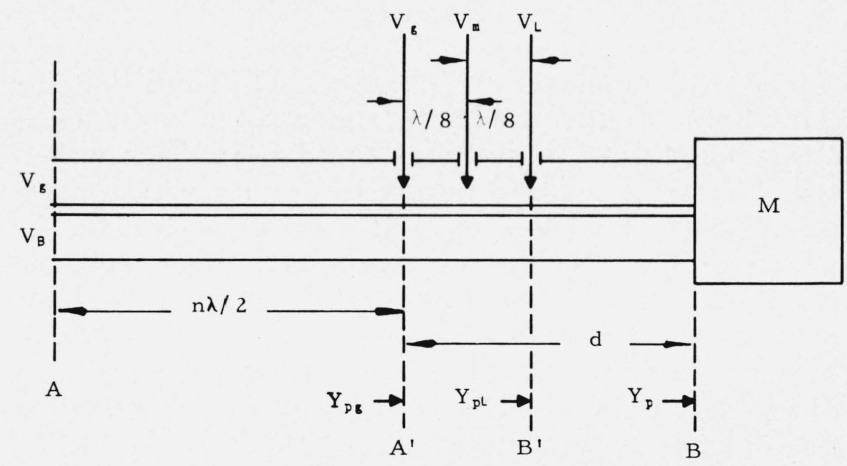

FigURE 7. Three-probe MP line for impedance and power measurements. 
calibration frequencies commonly furnished at present for commercial power meters may be obtained with one MP line.

While high-resistance disks are not yet available, low-resistance Bolovacs can be used in connection with feed-through power measurements in two ways:

(1) One way is to calibrate existing directional couplers and their associated side-arm power meters with the Bolovac. The power meters may be calibrated independently first with the Bolovac using the procedures described above. Coupling ratios may next be determined in a conventional manner employing one of the calibrated power meters to read the main-line net power and another meter to read the side arm net power. Bolovacs may, of course be used instead of power meters to measure these two powers.

(2) A second way is to calibrate a probe-type "voltmeter" in a slotted line or an MP line in terms of line voltage as a function of frequency. The procedure recommended in reference [5], can be used, where the known voltage is furnished by the Bolovac. The sinusoidal voltage distribution in a shorted slotted line section is used in that procedure to calibrate a "voltmeter." $P_{n}$ is then computed from the readings of this probe-voltmeter. The dynamic linearity of this probe type voltmeter may also be checked versus the sinusoidal voltage distribution in the line. Coupling factors between $P_{n}$ in the slotted line and the probe voltmeter of the order of 30 to $40 \mathrm{~dB}$ were obtained at the $\mathrm{Na}$ tional Bureau of Standards at frequencies of 4 to 12 $\mathrm{GHz}$ with a normal probe penetration (of about $25 \%$ of interconductor spacing). The dynamic range depends on the range of the power meter used with the probe. Commercial meters are available with ranges of a microwatt to $100 \mathrm{~mW}$ or a range of $50 \mathrm{~dB}$. This range may be further increased by employing attenuator pads in series with the probe power meter. An overlapping calibration range, with and without the pad, will assure range continuity. Thus voltage magnitudes and their ratios may be made available enabling the measurement of kilowatts of feed-through power during operation.

\section{Error Analysis, Dynamic Range, Results}

The error analysis of reference [1] shows that the total limit of uncertainty in the Bolovac voltages is \pm 0.1 percent at frequencies to $18 \mathrm{GHz}$. This uncertainty may be realized in any laboratory with a properly constructed Bolovac and associated bridge. As stated before, the Bolovac with its bridge needs no rf calibration. It is assumed that dc (or audio) voltage measuring capabilities required to measure bridge biasing voltages $V_{0}$ and $V_{1}$ for eq (7) with high accuracy are available, in which case the rf power absorbed by the Bolobac is as accurate as $P_{\mathrm{dc}}$ (sub).

In some applications of the Bolovac for power measurements additional sources of uncertainty are those contributed by (1) the slotted lines or MP lines needed to determine the resistive components of the load or of the rf-power-absorbing system and (2) the losses in auxiliary components such as the slotted lines, stub tuners, and connectors which are not a part of the specified load.

The limits of uncertainty in measuring resistive components of loads at the NBS are given in the references $[7,19]$. For frequencies up to $18 \mathrm{GHz}$ with $7 \mathrm{~mm} 50-\Omega$ slotted line techniques and precision LPC -7 connectors the uncertainties are

$$
\Delta S=1.002+0.002 \mathrm{f}(\mathrm{GHz}) .
$$

For mated configuration of latest improved type-N connectors these are

$$
\Delta S=1.004+0.004 \mathrm{f}(\mathrm{GHz}) .
$$

The uncertainties at lower echelon laboratories may be found in reference [19]. These depend on many factors including the quality of the facilities and competence of personnel. For $S$ values of 1.2 or less at frequencies to $8 \mathrm{GHz}$ the uncertainty in $R_{p}$ may be 1 percent or less. At $18 \mathrm{GHz}$ it may be as high as 3 percent. For higher $S$ values the uncertainty does not increase appreciably.

Power losses in stubs, slotted lines and connectors are of the order of 1 to 5 percent at frequencies to 18 $\mathrm{GHz}$ and can be determined with an uncertainty of 1 to 5 percent $[7,20]$.

As said before, component losses can be measured with the Bolovac or a precalibrated power meter. For example, the net power flow in a system may be measured with the Bolovac and the loss is given by the power drop of the power meter when the component is inserted between the slotted line and the meter. Or when the bolovac is used as an absorption power meter the maximum available (conjugately matched) power from a stable source is measured before and after the lossy component is inserted in the system. Allowances must be made for the effect of the magnitude of $S$ on the losses of components, particularly of stub tuners. For this purpose two absorption-type Bolovacs having widely different values of $R_{s}$ may be used with the same source of $P_{c}$.

The disk is the heart of the Bolovac. Though a number of commercial firms are making thin-film disk resistors, the NBS was to date not successful in locating a supplier of the needed disks having a sufficiently high temperature-coefficient of resistivity, and had to develop its own disks. A separate paper is planned to describe the in-house fabrication of the disks. Briefly the characteristics of those realized to date are as follows:

Film material-platinum, gold and carbon

Electrodes - gold in most cases

Dimensions - as required for $50 \Omega, 7 \mathrm{~mm}$ coaxial line

Resistance of -0.2 to $10 \Omega$ ( 0.4 to $20 \Omega$ per side) gold disk

units, a couple of $25-\Omega$ platinum units, and a number of $50-\Omega$ carbon units 
Substrate - glass, quartz, or 2-mil high-temperature plastic

Power dissipation (continuous) in the assembly-

0.25 to $0.5 \mathrm{~W}$. The maximum power dissipation is several times higher; however, the long-term resistance drift is greater as the power dissipation is increased. This drift does not interfere with the application of the Bolovac.

Incremental resistance (bolometric) approximately 1 to $5 \Omega$ per watt

Dynamic range of individual disks approximately $10: 1$ in voltage $(20 \mathrm{~dB})$ for precisions of the order of 1 to 2 percent. Precision increases rapidly as the range is kept narrower. The voltage at the low end is limited by the bridge stability and ratio of rf power to total bias power. At the high end it is limited by the ratio of the fractional bias power required to restore balance.

Maximum accuracy with an equal arm bridge is realized when the $\mathrm{rf}$ power is $2 / 3$ of the total power dissipated in the disk [21].

The range of power obtained so far in a $50-\Omega$ system is approximately 200 microwatts to half a watt at firstorder agreements with calorimetrically calibrated power meters of 0.5 to 5 percent at frequencies of $10 \mathrm{MHz}$ to $18 \mathrm{GHz}$ respectively. Efforts to optimize structural details, particularly of the disks, were limited. Voltages of $25 \mathrm{mV}$ at 1 percent uncertainty ${ }^{2}$ and of $300 \mathrm{mV}$ at 0.1 percent were measured at 10 $\mathrm{MHz}$ with Bolovacs using two thermistors instead of the usual disk. Experimental evidence indicates that one can measure $0.1 \mathrm{~V}$ with low resistance $(0.1$ to $0.2 \Omega$ ) disks with the same uncertainty. The advantages here are that the disks can be used to $18 \mathrm{GHz}$ as against $1 \mathrm{GHz}$ for the thermistors. Also the disks are much superior mechanically.

It can be shown that the effect of the antiresonant impedance of good sliding shorts shunting Bolovac disks of resistance values below $100 \Omega$ is negligible. Since higher resistance Bolovacs will hardly be considered for use as absorption power meters, there is no need dwelling on this effect.

One may wind up the discussion of the error analysis by pointing out the major sources of uncertainties in associated equipment. These are residual VSWR of slotted and MP lines, and the limited reproducibility caused by connector contacts and variations in their discontinuities. As frequencies increase, this problem is aggravated. The next largest source is the instability of rf source outputs when relatively long-time intervals (1 to $3 \mathrm{~min}$ ) are required to transfer an amplitude value from the Bolovac to a source like an rf micropotentiometer, particularly when accuracies better than 0.5 percent are needed. In the latter case it may be best to reduce the time element by coaxial switching.

There are numerous commercial bridges presently used with bolometric power mounts. These can be

${ }^{2}$ The uncertainty consists of a limit of systematic errors of 0.5 percent and a one-sigmavalue of 0.5 percent for random errors. used or adapted for use with Bolovacs. A high-precision miniaturized bridge recently developed at the NBS may be of interest for this purpose. [22]

\section{Conclusion}

The application of the Bolovac to standardize power meters and to measure power transmitted in TEM coaxial lines introduces a superior technique for all echelons of calibration and measurement. One of its major advantages is the elimination of the error contributed by impedance mismatch at the source and load ends of the transmission line. The work at the NBS so far was limited to $7 \mathrm{~mm}-50-\Omega$ coaxial lines and frequencies to $18 \mathrm{GHz}$. Mechanical precision of $14 \mathrm{~mm}-50-\Omega$ Bolovac disks (for frequencies to $9 \mathrm{GHz}$ ) should be higher than that of the $7 \mathrm{~mm}$ units. On the other hand $3.5 \mathrm{~mm}$ disks (for frequencies to $36 \mathrm{GHz}$ ) will probably be inferior by comparison. However, the application of the Bolovac to $36 \mathrm{GHz}$ appears feasible and desirable. One must not lose sight of the fact that the same Bolovac makes it possible to apply known voltages for experimental and practical treatment of problems other than power measurement at microwave frequencies $[4,5,6]$. For example, the Bolovac was recently used to measure deflection amplitude versus frequency of an oscilloscope to $18 \mathrm{GHz}$ for the first time in terms of voltage directly, instead of indirectly in terms of the time consuming and laborious method of measuring power and impedance.

Appreciation is hereby expressed to C. M. Allred and C. C. Cook for a constructive review of the paper, to P. E. Werner for his efforts in disk fabrication development, and to W. J. Blank and R. P. Chariton for performing countless painstaking observations and measurements.

\section{Appendix A. Net Power Flow in a Trans- mission line is $\left(V_{\max } V_{\min }\right) / Z_{0}$ (ref. 11, p. 564)}

Net power flow through plane $m$, figure 3 , is then

$$
\begin{aligned}
P_{n}=\frac{V_{m}^{2}}{R_{p}}= & \frac{V_{\max } V_{\min }}{Z_{0}}=\frac{V_{\max }^{2}}{S Z_{0}}=\frac{1}{S Z_{0}}\left(V_{m} \frac{V_{\max }}{V_{m}}\right)^{2} \\
& =V_{m}^{2} \frac{1}{S Z_{0}}\left(\frac{V_{\max }}{V_{m}}\right)^{2} \therefore R_{p}=S Z_{0}\left(\frac{V_{m}}{V_{\max }}\right)^{2}
\end{aligned}
$$

where

$V_{m}=\mathrm{rf}$ voltage in plane $m$

$V_{\max }=$ voltage maximum in a lossless coaxial line feeding power to the load M.

$R_{p}=$ reciprocal of the equivalent conductance of the load M at plane $m$. 


\section{Appendix B. Rf Voltage Measured by Bolovac}

In case of an equal-arm bridge the biasing power dissipated in the bolometer arm is

$$
P_{0}=\left(\frac{1}{2} V_{0}\right)^{2} / R_{m}
$$

where

$V_{0}=$ initial biasing voltage applied to the bridge at balance

$R_{m}=$ bias resistance of the Bolovac.

When rf is applied to the bolometer arm and the biasing voltage is reduced to rebalance the bridge

$$
P_{1}=\left(\frac{1}{2} V_{1}\right)^{2} / R_{m}
$$

where $P_{1}=$ biasing power after $\mathrm{rf}$ is applied.

The rf power dissipated in the bolometer arm is

$$
\left(P_{0}-P_{1}\right)=P_{\mathrm{rf}}=\frac{1}{4 R_{m}}\left(V_{0}^{2}-V_{1}^{2}\right)=V_{\mathrm{rf}}^{2} / R_{\mathrm{rf}}
$$

Let $R_{T 1}$ and $R_{T 2}$ be the resistance of the two halves of the disk respectively and let

$\alpha=\frac{R_{T 1}}{R_{T 2}} \geqslant 1$; then for $\alpha=1, R_{\mathrm{rf}}$ of the disk $=\frac{1}{4} R_{m}$ and

$$
\begin{aligned}
& 4 V_{\mathrm{rf}}^{2}=\frac{1}{4}\left(V_{0}^{2}-V_{1}^{2}\right) \\
& \therefore V_{B}=V_{\mathrm{rf}}=0.25\left(V_{0}^{2}-V_{1}^{2}\right)^{1 / 2} .
\end{aligned}
$$

One may read voltages directly across the Bolovac instead of across the bridge (employing a relatively high input-resistance dc voltmeter, e.g., a presently available four or five digit voltmeter). In this case for any bridge, let

$V_{B 0}=$ bias voltage drop across the Bolovac without $\mathrm{rf}$

$V_{B 1}=$ bias voltage drop across the Bolovac with rf $\Delta V_{B 0}=V_{B 0}-V_{B 1}$

$$
P_{\mathrm{rf}}=P_{B 0}-P_{B 1}=\frac{1}{R_{m}}\left[2 V_{B 0} \Delta V_{B 0}-\left(\Delta V_{B 0}\right)^{2}\right]
$$

and for $\alpha=1, P_{\mathrm{rf}}=\frac{4 V^{2}{ }_{\mathrm{rf}}}{R_{\mathrm{m}}}$

$$
V_{\mathrm{rf}}=\frac{1}{2}\left[2 V_{B 0} \Delta V_{B 0}-\left(\Delta V_{B 0}\right)^{2}\right]^{1 / 2} .
$$

For relatively large values of $V_{B 0} / \Delta V_{B 0}$, (say 5000:1),

$$
\left.V_{\text {rf }} \doteq \frac{1}{2}\left(2 V_{B 0} \Delta V_{B 0}\right)^{1 / 2} \quad \text { (to } 0.01 \%\right) .
$$

For $\alpha>1, R_{\mathrm{rf}}=\frac{\alpha}{(1+\alpha)^{2}}\left(R_{\mathrm{m}}\right)$

and

$$
V_{\mathrm{rf}}=\frac{\alpha^{1 / 2}}{(1+\alpha)}\left[2 V_{B 0} \Delta V_{B 0}-\left(\Delta V_{B 0}\right)^{2}\right]^{1 / 2}
$$

or for large $V_{B 0} / \Delta V_{B 0}$

$$
V_{\mathrm{rf}} \doteq \frac{\alpha^{1 / 2}}{(1+\alpha)}\left(2 V_{B 0} \Delta V_{B 0}\right)^{1 / 2}
$$

\section{Appendix C. Method of Adjusting the Uniformity of the Voltage Distribution in a Slotted Line for a $\mathbf{Z}_{0}$-Match}

1. The three probes (fig. 4) are located in succession at any one plane of the line. The gain of each associated VSWR(s) indicator is set to read the same as the others.

2. The three probes are then placed in the line spaced within a distance preferably of $\lambda / 4$. In case of physical space limitations one or two of the probes may be moved a distance $\lambda / 2$ from its originally intended position.

3. The stub tuner is adjusted for maximum output indication of the power meter, i.e., for a conjugate match. This procedure is useful because the generator impedances are reasonably close to $Z_{0}$ and thus a roughly approximate match is obtained.

4. The readings of the three $S$ indicators are then equalized by readjusting the stubs. In our experience it has been found very helpful to watch the power meter indication as the stubs were readjusted for the $Z_{0}$ match in order to prevent abrupt large changes in its reading.

The procedure suggested above was performed at the National Bureau of Standards by inexperienced personnel in a small fraction of the time required by a single-probe procedure.

\section{Appendix D. Measurement of Power With a Multiple-Probe Line}

Referring to figure 7 [18]

$$
\begin{aligned}
Y_{p g} & =G_{p g}+j B_{p g}=\frac{1}{R_{p g}}-j \frac{1}{x_{p g}} \\
P_{B} & =V_{g}^{2} G_{p g}
\end{aligned}
$$

$$
G_{p g}=\frac{V_{L}}{V_{g}} \frac{1}{Z_{0}}\left[1-\frac{1}{4}\left(2 \frac{V_{m}}{V_{L}} \frac{V_{m}}{V_{g}}-\frac{V_{L}}{V_{g}}-\frac{V_{g}}{V_{L}}\right)^{2}\right]^{1 / 2},
$$

$j B_{p g}=\frac{1}{j x_{p g}}=\frac{j}{2 Z_{0}}\left(1+\frac{V_{L}^{2}}{V_{g}^{2}}-\frac{2 V_{m}^{2}}{V_{L}^{2}}\right)$

$$
G_{p L}=\frac{V_{g}}{V_{L}} \frac{1}{Z_{0}}\left[1-\left(\frac{V_{L}}{2 V_{g}} \frac{V_{m}}{V_{L}} \frac{V_{m}}{V_{g}}+\frac{V_{g}}{2 V_{L}}\right)^{2}\right]^{1 / 2}
$$

$$
j B_{p L}=\frac{1}{j x_{p L}}=\frac{j}{2 Z_{0}}\left(1+\frac{V_{g}^{2}}{V_{L}^{2}}-\frac{2 V_{m}^{2}}{V_{L}^{2}}\right)
$$


where

$V_{g}$ is the voltage in the plane of the probe next to the generator. When the Bolovac is used as shown in figure $3, V_{g}=V_{B}$.

$V_{m}$ and $V_{L}$ are the voltages in the planes of the other two probes respectively.

The $p g$ subscripts identify impedance and admittance components in the plane of the probe closest to the source.

The $p L$ subscripts identify impedance and admittance components in the plane of the probe closest to the load M.

The above impedance equations are written in terms of voltage ratios to emphasize that only voltage ratio measurements are needed and are obtainable with greater accuracy than absolute voltages. The distance $B^{\prime}-B$, figure 7 , may be made equal to an integral number of half wavelengths to render $G_{p L}$ equal to that at any chosen input plane of the load, M. The power absorbed by the load must of course be computed in terms of a known voltage and input conductance in one and the same plane, e.g., in plane $A^{\prime}$, figure 7 .

\section{References}

[1] Selby, M. C., Bolometric voltage and current (Bolovac) standard for high and microwave frequencies, J. Res., Nat. Bur. Stand. (U.S.) (Eng. and Instr.), 72C, No. 1 (Jan.-Mar. 1968).

[2] Beatty, R. W., Macpherson, A. C., Mismatch errors in microwave power measurements, Proc. IRE 41, pp. 1112-1119 (Sept. 1953).

[3] Rumfelt A. Y., and Elwell, L. B., Radio Frequency Power Measurements, Proc. of IEEE 55, No. 6, Survey and Extensive Bibliography, p. 837 (June 1967).
[4] Selby, M. C., Electrothermic instruments for measuring voltage or current, Patent No. 3,487.305; December 30, 1969.

[5] Selby, M. C., Voltage measurement at high and microwave frequencies in coaxial lines, Proc. of IEEE 55, No. 6, 877-882 (June 1967).

[6] Beatty, R. W.. A microwave engineer's point of view toward voltage measurements, private communication (June 1967).

[7] Beatty, R. W., Coaxial power impedance and attenuation calibration methods at NBS. The Microwave Journal 111 , 65-75 (May 1968).

[8] Microwave power measurement, Hewlett-Packard Application Note, No. 64, HP AN 64.

[9] Personal communication with personnel of NBS and other laboratories.

[10] Government specification affecting calibration laboratories, National Conference of Standards Laboratories, April 1969. Report by A. E. Hess, RF Calibration System Analysis Project, NBS Boulder, Colorado, Project No. NBS 2720482, January 21, 1969.

[11] Ryder, John D., Networks, lines and fields, Second Edition, 1959 (Prentice-Hall, Inc.), p. 343

[12] International Telephone and Telegraph Corp., Reference data for radio engineers, Fourth Edition (1957), p. 574.

[13] NBS Monograph 96, Electrical parameters of precision coaxial, air-dielectric transmission lines, p. 14 (June 30, 1966).

[14] Sucher, M., and Fox, J., Handbook of microwave measurements, 1 (John Wiley \& Sons, New York), p. $122 \mathrm{ff}$.

[15] King. D. D., Measurements at centimeter wavelengths (Van Nostrand Series), pp. 197-199 (1952).

[16] Duffin, W. J., Three-probe method of impedance measurement, Wireless Engineer 29 (Dec. 1952), p. 317.

[17] Ginzton, E. L., Microwave measurements (McGraw-Hill Book Co., Inc., 1957), p. 236.

[18] An adaptation of the three-voltmeter method for measuring power and voltage at high frequencies, Electric and Musical Industries, Ltd., Hayes, Middlesex.

[19] IEEE Technical Committee Report on the State-of-the-Art of Measuring unbalanced transmission line impedances, R. L. Jesch, Chairman, June 3, 1970. (To be published.)

[20] Ragan, G. L., Microwave transmission circuits, R. L., Series No. 9 (McGraw-Hill Book Co., Inc., 1948), p. 150.

[21] Selby, M. C., Single-bolometer series-parallel circuit for accurate voltage and power measurement, Instrument Society of America, Preprint No. 21. 6-1-64, October 1964

[22] Larsen, N. T., and Clague, S. R., The NBS type II power measuring system, to be presented at the ISA, 25th Annual Conference, October 26-29, 1970.

(Paper 74C3 \& 4-306) 